\title{
Gain Recovery Dynamics and Photon-Driven Transport in Quantum Cascade Lasers
}

\section{Citation}

Choi, Hyunyong, Laurent Diehl, Zong-Kwei Wu, Marcella Giovannini, Jérôme Faist, Federico Capasso, and Theodore B. Norris. 2008. "Gain Recovery Dynamics and Photon-Driven Transport in Quantum Cascade Lasers." Physical Review Letters 100 (16). https://doi.org/10.1103/ physrevlett.100.167401.

\section{Permanent link}

http://nrs.harvard.edu/urn-3:HUL.InstRepos:41372672

\section{Terms of Use}

This article was downloaded from Harvard University's DASH repository, and is made available under the terms and conditions applicable to Other Posted Material, as set forth at http:// nrs.harvard.edu/urn-3:HUL.InstRepos:dash.current.terms-of-use\#LAA

\section{Share Your Story}

The Harvard community has made this article openly available. Please share how this access benefits you. Submit a story.

Accessibility 


\title{
Gain Recovery Dynamics and Photon-Driven Transport in Quantum Cascade Lasers
}

\author{
Hyunyong Choi, ${ }^{1}$ Laurent Diehl, ${ }^{2}$ Zong-Kwei Wu, ${ }^{1}$ Marcella Giovannini, ${ }^{3}$ Jérôme Faist, ${ }^{3}$ \\ Federico Capasso, ${ }^{2}$ and Theodore B. Norris ${ }^{1}$ \\ ${ }^{1}$ Center for Ultrafast Optical Science and Department of Electrical Engineering and Computer Science, University of Michigan, \\ Ann Arbor, Michigan 48109-2099, USA \\ ${ }^{2}$ School of Engineering and Applied Sciences, Harvard University, Cambridge, Massachusetts 02138, USA \\ ${ }^{3}$ Institute of Physics, University of Neuchâtel, CH-2000 Neuchâtel, Switzerland
}

(Received 12 May 2007; published 24 April 2008)

\begin{abstract}
Quantum cascade lasers are semiconductor devices based on the interplay of perpendicular transport through the heterostructure and the intracavity lasing field. We employ femtosecond time-resolved pumpprobe measurements to investigate the nature of the transport through the laser structure via the dynamics of the gain. The gain recovery is determined by the time-dependent transport of electrons through both the active regions and the superlattice regions connecting them. As the laser approaches and exceeds threshold, the component of the gain recovery due to the nonzero lifetime of the upper lasing state in the active region shows a dramatic reduction due to the onset of quantum stimulated emission; the drift of the electrons is thus driven by the cavity photon density. The gain recovery is qualitatively different from that in conventional lasers due to the superlattice transport in the cascade.
\end{abstract}

DOI: 10.1103/PhysRevLett.100.167401

PACS numbers: 78.47.- $\mathrm{p}, 42.55 . P x, 73.63 .-\mathrm{b}$

Electronic transport is central to understanding the physics of solid state devices such as transistors, detectors, modulators, light emitting diodes, and laser diodes. The discovery of quantum phenomena in nanometer-thick layers, including size quantization [1,2], the quantum confined Stark effect [3], and resonant tunneling [4] has greatly enriched semiconductor physics by enabling novel functionalities and devices. Here, we investigate the transport of electrons through a quantum cascade laser (QCL) [5]; QCL's are particularly interesting as tools for investigating electronic transport, as they operate under conditions of fully developed quantum transport that cannot be described in the framework of drift-diffusion equations.

In a QCL, electrons cascade through the heterostructure, ideally emitting one photon via stimulated emission in each active region. Thus, QCL operation results from a coupling of perpendicular drift transport with the intracavity optical field. In this Letter, we demonstrate remarkable changes in electronic transport across the device as the current is increased above threshold: from drift in the presence of phonon scattering to photon-driven transport via stimulated emission leading to a greatly reduced transit time across the entire length of the device. Our approach to studying the coupling of transport and laser dynamics is to time resolve the dynamics of the gain recovery following an impulsive perturbation of the intracavity field. Pumpprobe methods have previously been used to explore the possibility of coherent transport in resonant-tunnelling QC structures [6] under nonlasing conditions [7]. Here, we explore the gain dynamics of operating QCL's below and above threshold, and the measured dynamics is used to develop a model for the QCL level populations coupled to the cavity photon density rate equation. We show that the transport is driven by the photon density, which is qualita- tively different from previous photon-assisted tunneling studies [8,9] in which an external classical field opens a new channel for transport.

The lasers used in this study (Fig. 1) are based on a "diagonal transition" in real space [10]. A population inversion is present at all bias fields in these experiments; the cavity gain is controlled by voltage tuning of the oscillator strength through the linear Stark effect. The lowest state of the injector region serves as both the electron reservoir and also the upper lasing state. Laser action takes place between the upper state (level 2) and the lower state in the active region (level 1), which is emptied via tunneling into the superlattice. Before an electron can contribute to stimulated emission in the next active region (i.e., the portion of the cascade heterostructure in which the optical transition takes place), it must drift across the superlattice injector region.

In a simple picture, one can see how the current is driven by the laser photon density. In a cascade heterostructure, the current through a given level is given by $J=e N / \tau$, where $e$ is the electron charge, $N$ is the areal density, and $\tau$ is the state lifetime. Below threshold, the upper state lifetime is determined by (off-resonant) phonon-assisted tunneling (20-50 ps here). Above threshold, the relevant lifetime should become the stimulated emission lifetime $\tau_{\mathrm{st}}$.

Two QCL's were fabricated and processed with different cavity length and width: laser N-432 was $3.53 \mathrm{~mm}$ long and $12.5 \mu \mathrm{m}$ wide, operating at $5.3 \mu \mathrm{m}$, and laser N-433 was $2.41 \mathrm{~mm}$ long and $17.1 \mu \mathrm{m}$ wide, operating at $5.25 \mu \mathrm{m}$. Gain recovery dynamics were investigated using ultrafast degenerate pump-probe techniques, with the QCL operating in continuous wave at a constant voltage. Tunable ( 2.5 to $7.5 \mu \mathrm{m}$ ) midinfrared (IR) pulses were generated from 


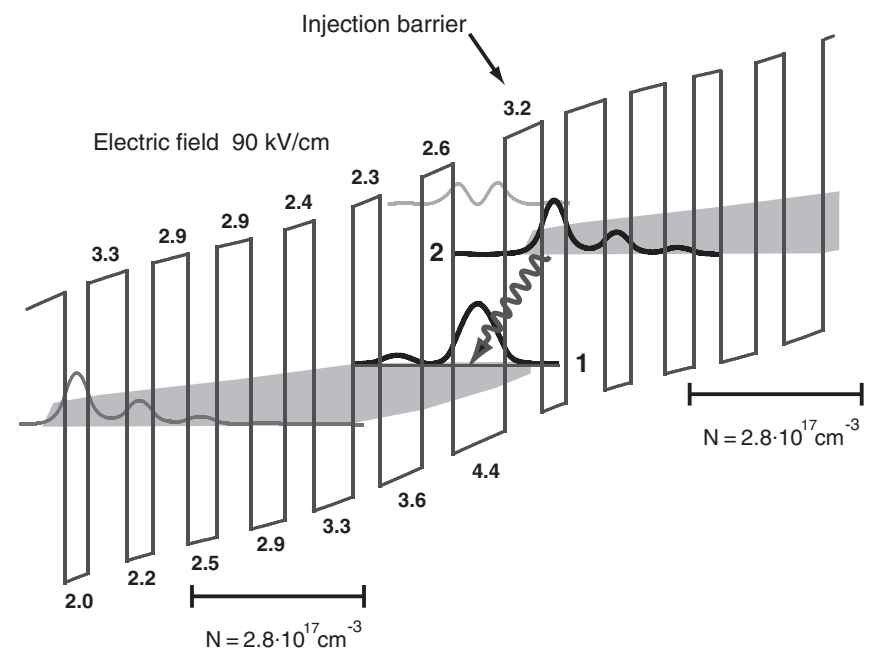

FIG. 1. Self-consistent band structure calculation of the $\mathrm{In}_{0.6} \mathrm{Ga}_{0.4} \mathrm{As} / \mathrm{In}_{0.44} \mathrm{Al}_{0.56} \mathrm{As}$ strained layers for two periods of the cascade heterostructure $(\mathrm{N}-432)$. Layer thicknesses are given in $\mathrm{nm}$. The wave functions for the upper (level 2) and lower lasing state (level 1) are shown, illustrating the diagonal nature of the transition in real space. The wavy arrow indicates the lasing transition. The miniband is indicated by the shaded gray region. The horizontal segments show the doped region for each period; $N$ is the donor density. Another QCL sample used in this experiment (N-433) with slightly different design parameters shows similar laser performance.

an Optical Parametric Amplifier and Difference Frequency Generator pumped by a $250-\mathrm{kHz}, 800-\mathrm{nm}$ Ti:Sapphire regeneratively amplified system. The mid-IR pulse width was typically $200 \mathrm{fs}$, which determines the temporal resolution of our differential transmission (DT) measurements. The pump and probe pulses were at the same wavelength (degenerate pump-probe), tuned to be resonant with the gain transition at each bias, coupled into the QCL waveguide (with polarizations $\pm 45^{\circ}$ with respect to the lasing polarization, respectively), and the DT of the weak probe pulse was measured following the saturation of the gain by a perturbing pump pulse. A $10-\mu \mathrm{m}$ spatial filter was positioned before the probe beam detection to isolate the portion of the probe coupled into the waveguide. The probe energy (40 pJ) was $1 / 20$ th the pump energy ( $800 \mathrm{pJ})$. In Fig. 2(a), selected bias-dependent DT results at $30 \mathrm{~K}$ are displayed. Carrier heating [11] due to the free carrier absorption of the pump was shown to be small compared to the stimulated-transition-induced gain depletion. For positive pump-probe delay, negative DT signals were observed at all bias currents. The recovery of the DT can be understood as the gain recovery due to electron transport in the cascade heterostructure following the pump-induced gain depletion.

The gain recovery was fit by solving the following threelevel, rate-equation model [12] including coupling to a single mode cavity photon flux: an example of the fit at $0.635 \mathrm{~A}$ is shown in Figure 2(b):

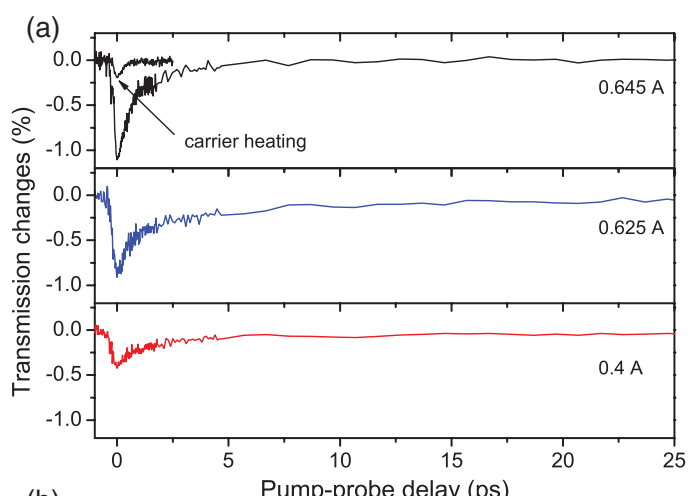

(b)

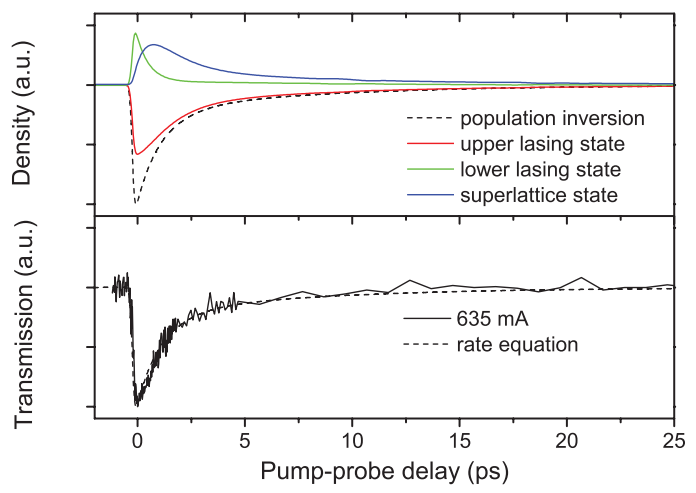

FIG. 2 (color). (a) Bias-dependent DT measurements of the gain recovery at $30 \mathrm{~K}$ for QCL from N-432. Carrier heating via TE-pump/TM-probe DT measurement is experimentally shown to be negligible. (b) Three-level rate-equation simulation of the population dynamics of upper state, lower state, and superlattice state (upper panel) and a fit to the the normalized DT signal at 0.635 A (lower panel) are displayed.

$$
\begin{aligned}
\frac{d S}{d t} & =\left[N_{p} \Gamma_{p} v_{g} g_{c}\left(n_{2}-n_{1}\right)-\frac{1}{\tau_{p}}\right] S+N_{p} \beta \frac{n_{2}}{\tau_{\mathrm{sp}}}, \\
\frac{d n_{2}}{d t} & =\frac{n_{\mathrm{SL}}}{\tau_{\mathrm{SL}}}-\frac{n_{2}}{\tau_{2}}-\Gamma_{p} v_{g} g_{c}\left(n_{2}-n_{1}\right) S, \\
\frac{d n_{1}}{d t} & =\frac{n_{2}}{\tau_{2}}-\frac{n_{1}}{\tau_{1}}+\Gamma_{p} v_{g} g_{c}\left(n_{2}-n_{1}\right) S, \\
\frac{d n_{\mathrm{SL}}}{d t} & =\frac{n_{1}}{\tau_{1}}-\frac{n_{\mathrm{SL}}}{\tau_{\mathrm{SL}}},
\end{aligned}
$$

where $n_{2}, n_{1}, n_{\mathrm{SL}}$ are the level 2, level 1, superlattice electron density, and $S$ is the photon density. $N_{p}(=25)$ is the number of stages in the QCL, $\Gamma_{p}$ is the confinement factor for one period ( $1.88 \%$, calculated), $v_{g}$ is the group velocity, $g_{c}\left(=\frac{4 \pi e^{2} z_{21}^{2}}{\epsilon_{0} n \lambda(2 \gamma) L_{p}}\right)$ is the gain cross section, $\tau_{p}$ is the photon lifetime, $\beta$ is the spontaneous emission factor (fraction of spontaneous emission emitted into the lasing mode), $\tau_{\mathrm{sp}}$ is the spontaneous emission lifetime $(\sim 1 \mu \mathrm{s}$, calculated), $\tau_{\mathrm{SL}}$ is the superlattice transport time, $\tau_{2}$ is the nonradiative lifetime of level 2 , and $\tau_{1}$ is the tunneling time from level 1 to the superlattice. We found that fits using fewer than three temporal components were unable to systematically fit the DT curves. 
As can be seen in the rate equations, there are four processes that enter the dynamics: (i) phonon-assisted relaxation out of the upper lasing state, (ii) stimulated emission out of the upper lasing state, (iii) depletion of the lower lasing state via tunneling, and (iv) transport across the superlattice.

The central result of this Letter is contained in Fig. 3(a) and $3(\mathrm{~b})$, which shows the upper state lifetime $\tau_{2}$, obtained from the rate-equation fits of the DT data versus bias current. Three processes contribute to the decay of the upper state: phonon-assisted intersubband relaxation [13] with time constant $\tau_{\mathrm{ph}}$, radiative decay by spontaneous emission $\tau_{\mathrm{sp}}$, and stimulated emission $\tau_{\mathrm{st}}$. The total upper state lifetime is given by $\tau_{2}^{-1}=\tau_{\mathrm{ph}}^{-1}+\tau_{\mathrm{sp}}^{-1}+\tau_{\mathrm{st}}^{-1}$. Since $\tau_{\mathrm{sp}}$ is several microseconds, radiative decay is negligible. Well below threshold, the upper state lifetime is essentially $\tau_{\mathrm{ph}}$, which is in the range of 20-50 ps. As the QCL bias voltage approaches threshold from below, stimulated transitions start to occur, reducing the upper state lifetime and increasing the current through the device. In Fig. 3(a) and 3 (b), a dramatic reduction in the upper state lifetime $\tau_{2}$ as
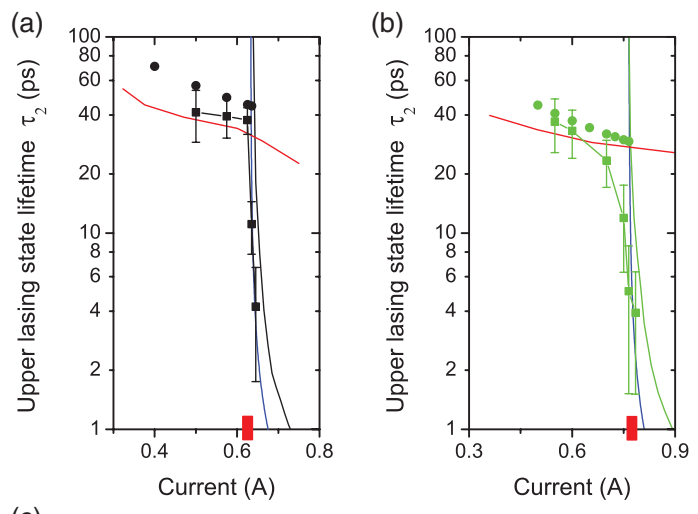

(c)

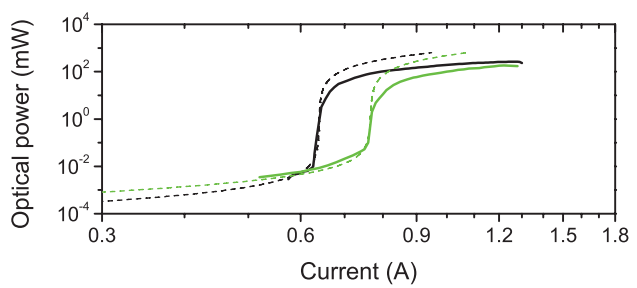

FIG. 3 (color). Bias-dependent level-2 lifetime $\tau_{2}$ is shown for $\mathrm{N}-432$ in (a) and N-433 in (b) (threshold is indicated by the red tick mark). The filled squares are $\tau_{2}$ obtained from rate-equation fits to the gain recovery DT data. The red solid lines in (a) and (b) are $\tau_{2}$ due to optical-phonon scattering calculated using the bias-dependent wave functions; the errors obtained by assuming monolayer fluctuations of the barrier width are around $10 \mathrm{ps}$. The filled circles are an estimate of the level-2 lifetime using $J=$ $e N / \tau_{2}$ and assuming the level-2 population $N$ is simply the doping density. The vertical blue, black lines in (a) and blue, green lines in (b) are explained in the text. (c) Steady-state rateequation fits (dashed line) to the measured $L-I$ curves (solid lines) are displayed. Black and green color are for N-432 and N433 , respectively. The only free parameter required to fit the $L-I$ curves with the rate-equation model is the $\beta$ factor. the QCL approaches threshold is very clear for both devices. Above threshold, $\tau_{\mathrm{st}}$ becomes of an order of a few ps and does not appear in the DT gain recovery dynamics (i.e., above threshold the DT dynamics are determined entirely by the lower state emptying and the superlattice transport components).

This behavior is in strong contrast to that of conventional atomic or molecular lasers, or of semiconductor lasers based on interband transitions; for those lasers, the gain recovery time constant is the spontaneous emission time $\tau_{\mathrm{sp}}$ below threshold, and (in the short cavity-lifetime limit) $\tau_{\mathrm{sp}} / r$ above threshold, where $r$ is the ratio of the pump rate to the threshold pump rate [14]. The QCL gain recovery curves in Fig. 2 and 3 show, however, an order-ofmagnitude speed-up in the upper state lifetime component of the gain recovery at threshold. The differences in gain recovery between QCL's and atomic, molecular, and interband semiconductor lasers can be traced to a combination of several unique features of QCL's.

The most important difference is the gain recovery in QCL's has a component that has no analogue in conventional laser systems, namely, the transport delay between active regions in the cascade structure. If this mechanism is removed from the rate equations, then those equations become the same as in conventional lasers, and the orderof-magnitude speed-up of the gain recovery just below threshold due to stimulated emission is not seen [15]. Each active region in a QCL is essentially an open system, coupled by transport through the superlattice to adjacent active regions; once an electron has made a downward transition via stimulated emission, it is not repumped into the excited state within the same active region. Instead, it must be transported to the next active region, with a corresponding delay. By contrast, stimulated emission in conventional lasers occurs in a closed system; following a downward transition, an electron is repumped into the excited state within the same atom or molecule.

The second contributing factor to the speed-up in gain recovery near threshold is the interplay of the nonradiative nature of the upper state decay far below threshold, and the turn-on of stimulated emission just below threshold. Well below threshold, the phonon-assisted lifetime is weakly bias-dependent. Just below threshold, the photon density in the cavity becomes of the order of a few hundred, which is sufficient to drive the stimulated emission lifetime down to a value comparable to the nonradiative lifetime. (Also, the $\beta$ factor of QCL's is rather high, approximately $10^{-3}$, as obtained from the $L-I$ curves). Above threshold, the stimulated lifetime continues to decrease as the current increases, but it no longer appears in the gain recovery dynamics (as the gain recovery is limited in that case by the lower state tunnelling and superlattice transport delays).

The self-consistency of the model used to understand the gain recovery was verified as follows. First, we calculated $\tau_{\text {st }}$ via the three-level rate-equation model [blue solid in Fig. 3(a) and 3(b)] as follows: the rate-equation model was 


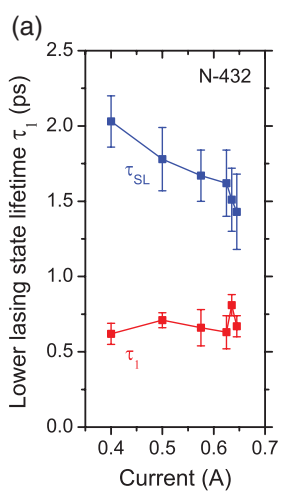

(b)

FIG. 4 (color). (a) Extracted time constants for N-432 from the rate-equation fit. $\tau_{1}$ is the level-1 lifetime (red), and $\tau_{\mathrm{SL}}$ is the time constant of gain recovery due to the superlattice transport (blue), as explained in the text. (b) Extracted time constants for N-433. $\tau_{1}$ and $\tau_{\mathrm{SL}}$ have same meaning as in (a). The error bars are determined from the chi-square values obtained in the rateequation fit.

used to fit the $L-I$ curves [dashed line in (c)], and the resulting $S$ was used to calculate $\tau_{\text {st }}$, and we found good agreement with the DT data. Second, we also calculated $\tau_{\text {st }}$ directly by obtaining the cavity photon density $S$ from the measured output power assuming $70 \%$ collection efficiency [solid line in (c)] and using $\tau_{\mathrm{st}}=N / v_{g} N_{p} g_{c} S$, where $N$ is the population inversion (again assumed to be the doping density), $v_{g}$ is the group velocity, $N_{p}$ is the number of cascading stages, and $g_{c}$ is the gain cross section; the result is shown as the black and green vertical line in Fig. 3(a) and 3(b), respectively. Finally, to check self-consistency, the rate equations used to fit the dynamics were solved in steady-state to obtain the threshold current and steady-state $L-I$ curves; these are seen in Fig. 3(c) to agree quite well with the experiment.

In addition to the upper state lifetime, two other components are obtained in fits to the gain recovery (Fig. 4) [16]. The fastest component $\tau_{1}$, approximately $0.7 \mathrm{ps,} \mathrm{corre-}$ sponds to the decay of the lower lasing state via tunneling (and is nearly independent of bias, consistent with estimations from the band structure). The second component, on the time scale of $2 \mathrm{ps}$, is attributed to the superlattice transport. We have observed this component in a variety of different QCL structures and have shown it can be understood as dielectric relaxation within the superlattice following the pump-induced perturbation [16]. It should be noted that because dielectric relaxation can be characterized by a single exponential process, the use of a single time constant in the rate equations for the superlattice may be justified.

In conclusion, time-resolved measurements of the gain recovery dynamics in QCL's show that the transport through the device and the cavity photon density are in- timately coupled, and that from just below to above threshold, the current through the device is determined by the stimulated emission rate into the lasing mode. We note that analogous tunnelling of electrons via a photon-assisted transition has been previously observed in resonanttunnelling devices under the application of an external oscillating electric field [8,9]. In those experiments, the presence of a strong external classical field opened up a new channel, increasing the transport across the device. In QCL's, the current near and above threshold is driven by the intracavity photon density, and the effect of quantum stimulated emission on transport at the few-photon level can be seen to turn on as the laser approaches threshold from below.

The authors would like to thank A. Belyanin and F.X. Kärtner for useful comments and discussion. This work was supported by US Army Research Office.

[1] R. Dingle, W. Wiegmann, and C.H. Henry, Phys. Rev. Lett. 33, 827 (1974).

[2] L. L. Chang, L. Esaki, and R. Tsu, Appl. Phys. Lett. 24, 593 (1974).

[3] D. A. B. Miller et al., Phys. Rev. Lett. 53, 2173 (1984).

[4] T. C. L. G. Sollner et al., Appl. Phys. Lett. 43, 588 (1983).

[5] J. Faist et al., Science 264, 553 (1994).

[6] F. Eickemeyer et al., Phys. Rev. Lett. 89, 047402 (2002); C. Sirtori et al., IEEE J. Quantum Electron. 34, 1722 (1998).

[7] R. C. Iotti and F. Rossi, Phys. Rev. Lett. 87, 146603 (2001); S. C. Lee, F. Banit, M. Woerner, and A. Wacker, Phys. Rev. B 73, 245320 (2006).

[8] A.H. Dayem and R. J. Martin, Phys. Rev. Lett. 8, 246 (1962); P. K. Tien and J. P. Gordon, Phys. Rev. 129, 647 (1963).

[9] P. S. S. Guimaräes et al., Phys. Rev. Lett. 70, 3792 (1993); B. J. Keay et al., Phys. Rev. Lett. 75, 4098 (1995); B. J. Keay et al., Phys. Rev. Lett. 75, 4102 (1995); K. Unterrainer et al., Phys. Rev. Lett. 76, 2973 (1996).

[10] J. Faist et al., Nature (London) 387, 777 (1997).

[11] M. Woerner, K. Reimann, and T. Elsaesser, J. Phys. Condens. Matter 16, R25 (2004). This paper investigates the role of hot carriers in QCL structures; our observation of the rapid reduction in upper state lifetime cannot be explained by hot carriers.

[12] Listed are parameters used in this work: full width at half maximum of transition $2 \gamma=28 \mathrm{meV}$ (measured), matrix element $z_{21}=0.3 \mathrm{~nm}$ (calculated), refractive index 3.17 (calculated), reflection coefficient 0.27 (calculated), waveguide loss $25 \mathrm{~cm}^{-1}$ (measured).

[13] D. Y. Oberli et al., Phys. Rev. B 40, 3028 (1989).

[14] A.E. Siegman, Lasers (University Science Books, Sausalito, California, 1986).

[15] H. Choi, Ph.D. thesis, University of Michigan, 2007.

[16] H. Choi et al., Appl. Phys. Lett. 92, 122114 (2008). 